\title{
Barriers to effective painless labor in developing countries and the remedial measures
}

\author{
Maj Gen (R) Professor Liaquat Ali, HI(M) \\ Head, Department of Anesthesiology, Pain Medicine \& Intensive Care, Fauji Foundation Medical College, Rawalpindi, Pakistan.
} Correspondence: Professor Liaquat Ali, HI (M); Phone: +923215836898

\section{Abstract}

Childbirth has been the most controversial subject in the pain management since ages. While there are some proponents which argue that pain is pain, and it's the physician's responsibility to relieve it as he does for the kidney pain or even simple headache; while some argue that it is not a pathological process, and that pain associated with labor brings about a stronger and lasting bondage between mother and child. A religious argument has also been presented against it. The introduction of epidural anesthesia revitalized interest in offering pain relief in labor even for prolonged periods. In resource poor countries, it has been looked upon with guarded skepticism. The scarcity of resources and staff, coupled with lack of a nation-wide awareness campaign has been largely responsible for the low practice of this modality in SAARC countries. Apicare Journal has raised this issue at SAARC level with the association of representative bodies of the member countries. This editorial is an attempt to present some insight on the barriers to painless labor and remedial measures in our countries.

Key words: Childbirth; Pain, labor; Pain relief; Epidural analgesia

Citation: Ali L. Barriers to effective painless labor in developing countries and the remedial measures. Anaesth. pain intensive care 2020;24(6):586-587; DOI: 10.35975/apic.v24i6.1395

Received: 22 November 2020, Reviewed: 22 November 2020, Accepted: 30 November 2020

American Society of Anesthesiology (ASA) states that

'There is no other circumstance where it is considered acceptable for a person to experience severe pain, amenable to safe intervention, while under a physician care'.

Labor is the most painful experience in a woman's life and unfortunately in developing countries, the practice of providing adequate pain relief during labor is limited to a few health care institutes.

Labor pain is not just a physical pain but it is also associated with fear, stress and anxiety which if uncontrolled can lead to poor outcome for both the mother and the baby ${ }^{1}$. Thus it is of utmost importance to provide painless labor to every parturient. The safest and most effective option for the painless delivery is local anesthetics via Lumbar Epidural Analgesia².

Antenatal period is an important time to discuss and encourage parturient about the option of painless delivery in the form of epidural analgesia (EA) but unfortunately the majority of women receive and rely on the information from family, friends, newspapers and magazines and least from a medical health professional. This leads to many misconceptions about EA such as the notion that EA leads to permanent backache, it has deleterious effects on the health of the baby and a strong belief that the parturients who opt for EA have high chances of ending up in either instrumental delivery or c-section. ${ }^{3}$

There is another factor that is related to our culture and society's conservative ideas where women believe that nature should be allowed to take its course and no interference be made in it. This notion is the result of the belief that only those parturient, who bears pain to deliver a child deserves to be called a true, good mother, while those who want painless delivery are somehow labelled as bad mothers.

The above-mentioned barriers are strongly related to the third big factor, which is the literacy and 
occupation of the parturient. It has been observed that knowledge about EA and its benefits was more among those who were graduates or working. On the contrary, house wives and undergraduates were least likely to opt for EA due to their lack of sound knowledge. ${ }^{4}$

Apart from these there is another factor that results in the reluctance of parturient in opting EA and that is the extra cost spent on the procedure, including the cost of the epidural kit and the services of a qualified anesthesiologist.

Next on the list are the obstacles presented by obstetricians in provision of painless delivery. Majority of the obstetricians felt that the nonavailability of an anesthetist was the main hindrance. Moreover, lack of trained staff and doctors for continual monitoring and to offer top-ups, adds to this factor.

Also, there is a fear among obstetricians that getting an EA will prevent the patient from bearing down during labor and ending up in operative deliveries. Painless labor has been associated with an increased risk of prolonging the labor and hence increasing the chances of operative deliveries. Although some obstetricians state that EA actually hastens the normal deliveries.

Lastly, few barriers that are related to the anesthesiologists; most common being the lack of expertise and the fear of procedure related complications. The time-consuming procedure followed by need of continual monitoring and ensuring effective analgesia requires effort on the part of the anesthesiologist, which may be difficult or even impossible if the anesthesia team is not well-staffed.

These barriers need prompt remedial measures as painless labor is the right of every woman going through child birth. There is a dire need to spread the awareness among our society about the benefits of EA for the mother and the baby and to remove all sorts of misconceptions that have been associated with painless labor for a long time.

Remedial measures may include regular antenatal visits and classes to be arranged with the purpose of discussing and preparing the parturient for EA under supervision of the anesthesiologist.

The fears of obstetrician about the non-availability of an anesthesiologist after the insertion of epidural catheter and the need for continual monitoring can be done by forming a dedicated hospital team which includes 2-3 staff nurses trained in handling EA during labor, headed by an expert anesthesiologist, which should be available for 24 hours a day, exclusively for providing painless labor services.

Moreover, Anesthesiology department can provide published detailed pamphlets on painless labor to the parturients in their third trimester, during their antenatal visits.

Painless Labor Day or week should be celebrated throughout the community with seminars being held by teams of anesthesiologists, gynecologists/obstetricians and pediatricians, which should include the participation of expectant mothers and provide them a platform to discuss their fears and clarify misconceptions regarding EA.

\section{Conclusion}

There have been many barriers to wide-spread acceptance of painless labor in developing countries. Misconceptions/myths among parturients, their mothers/mothers-in-law, and even obstetricians, have been the main obstacles. A lack of adequate number of trained anesthesiologists and pain nurses is also a big factor.

There is a strong need to address the misconceptions/myths; to ensure availability of dedicated trained staff, and create awareness among the parturients on a priority basis.

\section{Conflict of interest}

The author has no conflict of interest, and the information and the opinions expressed in this editorial are entirely based upon the author's vast experience.

\section{References}

1. Rao ZA, Haq ME, Ali L. Evaluation of the analgesic and adverse effects of tramadol in combination with low dose bupivacaine for painless epidural delivery. Pak Armed Forces Med J 2010;60(3):415-9 [Free full text]

2. Khan KS, Khan BF, Rasul S, Chohan U. The safety of epidural analgesia in labour and its effect on delivery- a case control study done in Pakistani women. J Pak Med Assoc 1993 Jun;43(6):115-7. [PubMed] [Free full text]

3. Minhas MR, Kamal R, Afshan G, Raheel H. Knowledge, attitude and practice of parturient regarding epidural analgesia for labour in a university hospital in Karachi. J Pak Med Assoc 2005;55(2):63-6. [PubMed] [Free full text] 